\title{
Knee stability and functional outcome following arthroscopic acl reconstruction: comparison between two different femoral tunnel positions
}

\begin{abstract}
Background and purpose: Anterior cruciate ligament (ACL) reconstruction is one of the most commonly procedure which are done by orthopedic surgeon. The aim of this study was to compare knee stability and functional results in two different tunnel position groups.

Materials and methods: In the current study, 472 patient s documents with ACL tear which were scheduled for reconstruction existed. By exclusion criteria, during the period January 2000 to July 2003, 159 total participants (147 men, 12women) were enrolled and were classified non-randomly in 2 groups by the femoral tunnel position which all of them were operated arthroscopically assisted ACL reconstruction.

Results: In our study 159 patients (147 male and 12 female) were participated. In the first group which femoral tunnel were placed at 11 o'clock position (right knee) and 1 o'clock (left knee), we found 28 cases with normal and 53 cases with varus and in the second group which femoral tunnel were placed at 10 o'clock position (right knee) and 2 o'clock position (left knee), we find 32 cases with normal and other 46 patients with varus mal alignment.

Conclusion: We found no significant difference between the 10 and 11o'clock femoral tunnel placement position when compared by IKDC and Lysholm score. Other point that we should mention was that there was significant difference in anterior tibial translation between two groups at 25 degree flexion (P value 0.03). Finally, femoral tunnel placement change was not an accurate indicator of functional outcome and knee laxity in our study.
\end{abstract}

Keywords: Anterior cruciate ligament Reconstruction, Knee stability, Femoral tunnel Placement, Lysholm scale, Surgeons
Volume 5 Issue 2 - 2016

\author{
Mohammad Razi, ${ }^{2}$ Salman Ghaffari,' Seyed \\ Mohamad Mehdi Daneshpoor ${ }^{3}$ \\ 'Department of orthopedic and sport medicine, Iran University \\ of medical science, Iran \\ ${ }^{2}$ Department of orthopaedic surgery, Mazandaran University of \\ medical science, Iran \\ ${ }^{3}$ Orthopaedic research center, Iran
}

\begin{abstract}
Correspondence: Salman Ghaffari,Assistant professor of orthopedic surgery, Mazandaran University of medical science, Orthopaedic research center, sari, Iran
\end{abstract}

Email orthosalman@yhoo.com

Received: May 05, 2016 | Published: June 13, 2016

\section{Introduction}

Arthroscopic Anterior Cruciate Ligament (ACL) reconstruction is one of the most commonly procedure which are done by orthopedic surgeons, ACL is one of the most commonly disrupted ligaments in the knee injuries and is a common cause of knee instability. ${ }^{1-3}$

This ligament consists of two functional fiber components. These are named by the site which they attach on the tibia, the anteromedial and posterolateral bundle. With knee extension, the posterolateral one is tight and the anteromedial bundle is relaxed. Since ACL deficient knee causes limitation to activity because of symptomatic instability and high prevalence of knee osteoarthritis, the most common method of treatment in these injuries is an ACL reconstruction. ${ }^{4,5}$ In some literature the estimated failure rate after this surgery reports approximately $10 \%$.

The probable problems of the arthroscopic reconstruction procedure included graft failure, femoral and tibial tunnel mismatch, interference screw fixation divergence, and posterior cortex destruction, graft laceration by the screw threads and graft impingement by nonanatomic tunnel placement.

Some literature has revealed that there is no significant difference in mid-terms follow-up results in functional and radiographic scores between one or two incision approach, but they didn't compare the placement of femoral tunnels. In the recent reports they showed that Anatomical ACL graft positioning seems to be an important factor for postoperative knee function and stability in ACL reconstruction. ${ }^{1,4}$

On the other hand, since the location of femoral tunnels for ACL replacement grafts remains a subject of debate. ${ }^{6}$ so, we conducted this study by the aim of knee stability evaluation and functional outcome following ACL reconstruction by two different femoral tunnel positions.

\section{Materials and methods}

In this prospective experimental study, the patients were referred to 3 referral orthopedics and trauma centers because of ACL tear which need to surgery. Between January 2000 to July 2003, 472 documents of ACL tear which were reconstructed arthroscopically assisted were recorded in these centers. Subjects were excluded if follow up were less than 1 year, patients with multiple ligament injuries, fractures, incomplete intraoperative data and any previous injury to the involved or contralateral knee or bilateral subjects and patients didn't refer for follow up for any reason. Finally 159 total participants [147 men $(92 \%), 12$ women $(8 \%)]$ were enrolled in the current study.

All ACL reconstructions were performed arthroscopically by one single experienced surgeon and under general anesthesia with a tourniquet applied to the proximal thigh during this mentioned period.

All participants were classified non-randomized to 2 group based on the femoral tunnel placement, group 1 ( $\mathrm{n}=81$, mean age: 26.32 years) which their femoral tunnel position was at the 11 o'clock (right knee) or 1 o'clock position (left knee) and group 2 ( $n=78$, mean age: 25.19 years) which their femoral tunnel position was at the 10 o'clock (right knee) or 2 o'clock position (left knee). On the other hand, this classification was not randomly because of first group were designed before the study had started as their femoral tunnel position was at 11 o'clock (right knee) or 1 o'clock position (left knee). Then, after these patients were operated the surgeon decided to operate other patients 
as the second group which their femoral tunnel position was at the 10 o'clock (right knee) or 2 o'clock position (left knee) because of studies were done in cadaver which showed better results with this position.

Before the study, all of the patients were rehabilitated based on the group 1 protocol. This group information was clear at the baseline and we extracted this information as a prospective study. On the other hand, at the second phase we conducted the data for second group as a prospective one. We examined our patients either with manual physical and either KT 1000 arthrometer and values were recorded.

All patients were operated in the supine position under general anesthesia. After the knee was placed in a leg holder with a tourniquet inflated to $350 \mathrm{mmHg}$, a diagnostic arthroscopy was performed with a 30 degree arthroscope. All of intra-articular lesion was assessed. If present, all meniscal pathology was treated with meniscal repair or partial meniscectomy before the reconstruction surgery. Cartilaginous lesion if possible treated by drilling. If needed to do graft, we operated them on the later time. Ipsilateral hamstring (semitendinosous \pm gracilis) or Bone patellar tendon bone auto graft harvest was done. A notchplasty was performed if the notch was stenotic. The ligament remnants free ends were debrided to their footprints on the tibia and the femur. If partial ligament injury was present it was remained unless became impinged. While the graft was prepared by assistant, the transportal femoral tunnel created by guide pins placement freehandly which should be placed at the posterior half of posterior lateral femoral condyle $(1,11$ and 2,10$)$ but should be at least $2 \mathrm{~mm}$ far farm posterior cortex after reaming. Then tibial tunnel on the guide was created from smaller size $(6 \mathrm{~mm})$. After reaming guide pins placed in the direction of femoral tunnel so that tibial tunnel should be along with femoral tunnel in the 90 degree knee flexion to prevent killer curve at the tunnel opening. After preparation of graft and placement in the suitable position, fixed by screw (BPTB) or endobutton (quadruple hamstring) or press-fit (BPTB). After irrigation and hemovacuum draining insertion, wound closure was done routinely. Knee immobilizer applied and the patient transferred to recovery room. A day post-surgery and after quadriceps strengthening exercise and range of motion training, the patient was discharged. In all patient physiotherapy was done by the same method but in the different centers because of referring them from different cities. We called all of them for evaluation and follow up. From these whom visited for follow up, physical examination and assessment by KT-1000 based on the Lysholm and IKDC were done.

Patients follow up was done and recorded by subjective assessment and physical examination (Lachmann, anterior drawer test and Pivot shift test) and also KT-1000.Prioperativecomplications were recorded separately.

On the other hand, Knee stability was measured using the Lysholm scale, which includes pain, subjective instability, use of a walking device, articular blocking, effusion and claudication when walking, ability to climb stairs, and difficulty in kneeling. The Lysholm point system is classified to (excellent, 95 to 100; good, 94 to 84; regular, 83 to 65). The International Knee Documentation Committee (IKDC) form was also used to evaluate the clinical outcome of the ACL reconstruction.

Statistical analyses were performed with SPSS version 18.00. We used chi-square and T-test analysis to evaluate overall differences in the deviation of bone tunnel positions from the referenced value between 2 groups. Significance was set at $\mathrm{P}<0.05$.

\section{Results}

In our study, only 159 of 472 total participants were included because the mentioned exclusion criteria. Of these 159 patients, 147 males $(92 \%)$ and 12 females (8\%) with the mean age of 25.76 years were participate. Based on the femoral tunnel placement patients were classified to two groups. In group 1,81 patients (76 male, 5 female) $(50.9 \%)$ with the mean age of 26.32 years and in group 2,78 patients ( 71 male, 7 female) $(49.1 \%$ ) with the mean age of 25.19 years were participated. In 75 patients left knee were involved and other 84 patients, right knee were involved.

Based on the mechanism, the most common one was football (94 patients, 59.1\%). others include (17participants, 10.7\%) martial Arts, wrestling (10 cases, $6.3 \%)$, car accident ( 8 cases, $5 \%$ ), basketball ( 7 cases, $4.4 \%$ ), volleyball (4 cases, $2.5 \%$ ) (Table 1 ).

Waiting time based on IKDC score was acute (0-2 weeks), sub acute (2-8 weeks) and chronic (more than 8 weeks). In our study, 35 subjects $(22 \%)$ were placed in the sub acute group and 124 patients (78\%) were placed in the chronic group. In 60 patients we found normal alignment and other 99 patients showed varus alignment. Based on the knee alignment, in the first group we found 28 cases with normal and 53 cases with varus and in the second group we find 32 cases with normal and other 46 patients with varus malalignment (Tables 2-4).

Based on the graft selection, we used bone patellar bone graft in 110 cases $(69.2 \%)$, quadruple hamstring in 43 patients $(37 \%)$ and quadruple semintendinosus in 6 cases $(3.8 \%)$.

Table I Patient demographics.

\begin{tabular}{ll}
\hline Characteristic & Study Population \\
\hline Number of Patients & 159 \\
Mean age & 25.7 \\
Gender & 147 men, I2 women \\
Side affected (percent) & 84 right (52.8\%) 75 left (47.25) \\
& 94 football, I7 martial arts, 10 wrestling, 8 car \\
Mechanism of injury & accident, 7 basketball, 5 job-injury, 4 volley ball, \\
& 3 falling, 3 ski, 2 climbing, I running \\
Mean time to surgery by & 20.79 (I-96) \\
month (range) &
\end{tabular}

Table 2 IKDC score.

\begin{tabular}{llllll}
\hline IKDC Score & & & & & \\
ROM* & & Frequency & Percent & $\begin{array}{l}\text { Valid } \\
\text { Percent }\end{array}$ & $\begin{array}{l}\text { Cumulative } \\
\text { Percent }\end{array}$ \\
\hline \multirow{5}{*}{ Knee Ligaments } & Valid A & 148 & 93.1 & 93.1 & 93.1 \\
& B & 10 & 6.3 & 6.3 & 99.4 \\
& C & 1 & 0.6 & 0.6 & 100 \\
& Total & 159 & 100 & 100 & - \\
& Valid A & 92 & 57.3 & 57.3 & 57.9 \\
& B & 48 & 30.2 & 30.2 & 88.1 \\
& C & 19 & 11.9 & 11.9 & 100 \\
& Total & 159 & 100 & 100 & - \\
& Valid A & 23 & 14.5 & 14.5 & 14.5 \\
& B & 117 & 73.6 & 73.6 & 88.1 \\
& C & 16 & 10.1 & 10.1 & 98.1 \\
& D & 3 & 1.9 & 1.9 & 100 \\
& Total & 159 & 100 & 100 & \\
& Valid A & 59 & 37.1 & 37.1 & 37.1 \\
& B & 91 & 57.2 & 57.2 & 94.3 \\
& C & 8 & 5 & 5 & 99.4 \\
& D & 1 & 0.6 & 0.6 & 100 \\
& Total & 159 & 100 & 100 & \\
& & & & &
\end{tabular}


Table Continued...

\begin{tabular}{llllll}
\hline $\begin{array}{lllll}\text { IKDC Score } \\
\text { ROM* }\end{array}$ & & Frequency & Percent & $\begin{array}{l}\text { Valid } \\
\text { Percent }\end{array}$ & $\begin{array}{l}\text { Cumulative } \\
\text { Percent }\end{array}$ \\
\hline Final Outcome & Valid A & 86 & 53.5 & 53.5 & 53.5 \\
& B & 54 & 34 & 34 & 87.4 \\
& C & 20 & 12.6 & 12.6 & 100 \\
& Total & 159 & 100 & 100 & - \\
Pivot Shift Test & Valid A & 130 & 81.8 & 81.8 & 81.8 \\
& B & 26 & 16.4 & 16.4 & 98.1 \\
& C & 2 & 1.3 & 1.3 & 99.4 \\
& D & 1 & 0.6 & 0.6 & 100 \\
& Total & 159 & 100 & 100 & \\
\hline
\end{tabular}

A: Normal, B: Near normal, C:Abnormal, D: Severe abnormal

*: Range of motion

Table 3 Lachmann test,Anterior drawer test

\begin{tabular}{llllll}
\hline & & Frequency Percent & $\begin{array}{l}\text { Valid } \\
\text { Percent }\end{array}$ & $\begin{array}{l}\text { Cumulative } \\
\text { Percent }\end{array}$ \\
\hline $\begin{array}{llllll}\text { 25 degree } \\
\text { Flexion }\end{array}$ & Valid B & 94 & 59.1 & 59.1 & 59.1 \\
& C & 49 & 30.8 & 30.8 & 89.9 \\
& D & 16 & 10.1 & 100 & 100 \\
70 degree & Total & 159 & 100 & 100 & - \\
Flexion & Valid B & 94 & 59.1 & 59.1 & 59.1 \\
& C & 53 & 33.3 & 33.3 & 92.5 \\
& D & 12 & 7.5 & 7.5 & 100 \\
& Total & 159 & 100 & 100 & \\
& ATT(mm) & Healthy & First & Second & P value \\
& Knee & Group & Group & \\
& Flexion & 5 & 8.3 & 7.2 & 0.03 \\
& 70 degree & 5.9 & 8.1 & 7.9 & 0.42 \\
\hline
\end{tabular}

Table 4 Meniscus tears frequency

\begin{tabular}{lllll}
\hline & Right & & Left & \\
Percent & Frequency & Percent & Frequency & Percent \\
\hline 1.3 & 2 & 18.2 & 29 & Valid C \\
65.4 & 104 & 39 & 62 & $\mathrm{~N}$ \\
18.2 & 29 & 25 & 25 & $\mathrm{P}$ \\
7.5 & 12 & 24 & 24 & $\mathrm{R}$ \\
7.5 & 12 & 19 & 19 & $\mathrm{~S}$ \\
100 & 159 & 100 & 159 & Total \\
\hline
\end{tabular}

N: Normal, P: Partial meniscectomy, C: Complete meniscectomy, R: Repair or abrasion, S: Subtotal meniscectomy

We used Bio-absorbable screw in 47 subjects $(29.6 \%)$, Titanium interference screw 97 cases (61\%), Transfix in 14 cases $(8.8 \%)$ and press-fit without instrumentation in only 1 patient $(0.6 \%)$.

Before ACL tearing all of our patients were in functional level 1 (severe) and after ACL tear they were placed in the 4 (sedentary) or 3 (poor) level of activity. We found no change in functional activity after the reconstruction in 94 cases (59\%). Other 65 patients (41\%) changed their functional activity. We should mention that all of these patients which changed their activity level were placed in the level of 3 (15 cases) or 2 (50 cases) or higher level in the interval time between injury and reconstruction. We didn't find significant difference in activity level between before and after reconstruction procedure (P value 0.42 ). On the other hand, reconstruction didn't effect on the activity level of our patients.
In pivot shift test, 130 cases were placed in the group A (81.8\%), 26 cases in group B $(16.4 \%)$ (glide+), 2 patients in group C $(1.3 \%)$ (Clunk $2+$ ) and only 1 case in group D (0.6\%) (Gross $3+$ ). Meniscus position was showed in the Table 3.

As a complication after the procedure, we found hemarthrosis which required to be evacuated in 2 patients $(1.3 \%)$, impingement required repeated arthroscopy and Cyclops debridement in 8 cases $(5 \%)$, ACL re-rupture in 1 patient $(0.6 \%)$ and only 1 case with septic arthritis which treated with arthroscopy, debridement and intravenous and oral antibiotic. Overall complication rate was $7.6 \%$. Of these complications, impingement was more common.

More frequent morbidity at donor site were seen in the bone patella tendon graft and less frequent were seen in an isolated semitendinosous graft. This variable had significant difference between them.

In 16 patients, we find knee laxity (side to side difference more than $3 \mathrm{~mm}$ ) which 9 cases were in the 11 o'clock femoral runnel placement and 7 others were placed in the 11 o'clock femoral tunnel position.

Anterior Tibial translation (ATT) in healthy knee by KT1000 at 25 degree was $5 \mathrm{~mm}$ and in 70 degree flexion was $5.9 \mathrm{~mm}$. These values in the second group at 25 degree were $7.2 \mathrm{~mm}$ and in 70 degree flexion were $7.9 \mathrm{~mm}$. ATT in the first group at 25 degree was $8.3 \mathrm{~mm}$ and in 70 degree flexion was $8.1 \mathrm{~mm}$. Analysis showed that there was significant difference between two groups at 25 degree flexion (P value 0.03 ) but there was not this difference at 70 flexion (P value 0.42 ) (Table 3 ).

There was more significant difference between two groups in knee laxity assessment tests such as Lachmann and Pivot shift test. ATT in second group in Lachmann test was lower than first group. We should mention that femoral tunnel placement at $10: 30$ or $10(1: 30$ or 2) o'clock causes function as same as posterolateral bundle of ACL. Indeed, this bundle in knee extension was extended and there was no laxity in Lachmann test while tunnel position at 11 (1) o' clock simulated.

\section{Discussion}

In current study, we assessed functional outcome following ACL reconstruction by two different femoral positions placed at the 11 and 10 o'clock. 472 patients participate in the study but after exclusion criteria finally 159 patients were included in our study.

There was no significant difference between the involved limb (right knee 84, left knee 75). Because of the low number of athletic patients which referred to us, we couldn't assess the relation between the involved knees based on the sport fields.

The most common injury was seen in the football because of our patients interested in this sport field and better support from federation. This result was similar to other surveys which showed that football was more frequent mechanism in ACL injury. ${ }^{1,7}$ The job-injury or car accident rate was $10 \%$.

Authors believe that tunnel position effect on the anterior cruciate ligament reconstruction result and this correlation is more significant in femoral tunnel rather than tibial tunnel. Rayan, Kaseta, revealed the importance of tunnel placement. ${ }^{8-11}$ These studies focused on the anteroposterior position of femoral tunnel.

The mean time between injury and refer to our clinic was 20.79 months. On the other hand, most of patients were referred late. So the patients were operated when he or she lost the knee performance 
widely, they couldn't perform the usual functional level before the operation and also knee laxity complications such as further instability events, DJD, meniscal tearing was inevitable. Thus, these subjects had serious impact on the final knee function and post operation results. ${ }^{12,13}$

Limb alignment in our patients was almost in varus (99 patients). We could mention that ACL tear was more common in patients with varus alignment in their limb.

It should be noted that more significant difference was seen between two groups by Lachmann and pivot shift test. Anterior Tibial translation (ATT) in Lachmann test at 25 degree was lower than the first group because femoral tunnel placement at 10:30 or 10 o clock caused similar function to posterolateral bundle of ACL. On the other hand, this bundle could be tight at knee extension thus may be translates lower. These results was against the study be Loh JC et al. ${ }^{10}$ because they showed there was no significant difference in the ATT between these tunnel positions, except at 90 degrees of knee flexion. ${ }^{10}$ Markolf KL believed that AP positioning of the femoral tunnel at the 11 o'clock position is more critical than $10 \mathrm{o}$ 'clock positioning in terms of graft force and knee laxity features at the of ACL reconstruction. ${ }^{13}$

Authors such as Loh et al. ${ }^{10}$ Muller et al. ${ }^{14}$ find that moving the femoral tunnel position from the 11 or 11:30 o'clock positions to 10 or 10:30 o'clock positions have better knee stability and function. ${ }^{10,14}$

Also others showed cadaveric study suggest that placing the femoral tunnel at the 10 o'clock position could improve rotatory knee stability compared with the 11 o'clock position. Both femoral tunnel positions were found to be effective at stabilizing the knee under anterior tibial load. However, when the knee is subjected to combined rotatory loads, the 10 o'clock position is better than the 11 o'clock position; yet neither femoral tunnel position could completely restore the kinematics and the in situ forces to the level of the intact knee. Thus, in isolation, replacing the AM or PL bundle of the ACL alone does not restore the complex function of the intact ACL. Recently, separate cadaveric studies from our research center as well as others, have shown that an anatomic ACL reconstruction replacing both the $\mathrm{AM}$ and PL bundles could more closely reproduce knee kinematics and in situ force in the ACL graft to the level of the intact knee thus, this current study confirms that while the current ACL reconstruction procedures have limitations, moving the femoral tunnel more laterally may have some advantages. ${ }^{16-17}$

According to the IKDC evaluation, there was no significant difference in our patients which may be caused by short term follow op period in our study. Using the IKDC score, $53.5 \%$ of cases placed in the good or near normal group. Study by Kartus et al. ${ }^{17} 74 \%$ of patients was classified as normal and near normal group. Also there was no significant difference between 2 groups using Lysholm score that may be caused by the short term follow op too. ${ }^{17}$ Mean score in the first and second group was 81 and 78 respectively and finally $54.1 \%$ of our patients reach to excellent or good score which was lower that literature such as khalfayan whom showed $69 \%$ of patients received this score. ${ }^{11}$

These finding is despite the study be Loh JC et al. ${ }^{10}$ who demonstrated the $10 \mathrm{o}$ 'clock position more effectively resists rotatory loads when compared with the 11 o'clock position. ${ }^{10}$

Despite the long time between injury and ACL reconstruction, $59 \%$ of our patients returned to activity as level as before the operation but others $(41 \%)$ didn't continue their previous sport fields which caused by fear of re-rupture and additional knee injury. Although this change was done but often they were able to do routine tasks in an ordinary fashion except sport. No patient was forced to change the job because of his/her knee function. This result was despite the study by Kannus et al. ${ }^{18}$ that showed that $80 \%$ of their patient finally changed their job because of ACL tear. It should be mentioned that their follow up period (8years) was longer that our study and they try conservative treatment for their patients. Finally they recommended no conservative treatment for complete tear because of this result and also characteristic osteoarthritic changes which may be seen in these patients after long term follow up..$^{18}$

\section{Complications}

Most frequent complication in our study was impingement ( 8 cases, $5 \%$ ) which referred by limited range of motion, pain and popping and may be caused by unsuitable tibial tunnel placement at anteroposterior aspect. We find only 1 case with peroneal nerve injury which was seen in the Simultaneous ACL reconstruction and lateral meniscus repair. In the study by Small et al, they revealed overall complications $1.8 \%$ in knee arthroscopic procedures and 2\% in isolated ACL reconstruction without neurovascular injury. Other neurovascular injury was seen in saphenous nerve followed by meniscus repair. Complications were more frequent than Small study.

We saw only one case $(0.6 \%)$ with complete tear although more than $3 \mathrm{~mm}$ ligament laxity was seen in 16 cases $(10 \%)$ which were 9 cases in the first group and 7 cases in the second group. Failure rate of graft in the different literature reported between $7-8 \%$ that may be similar to our study if we consider the ligament laxity.

\section{Limitation of the study}

One of our limitation was the lack of archives in some cases includes wrong telephone number or address which caused access to patients unreachable. In some cases, the patients were to be far from or center and this cause they didn't trend to be visited despite our insistent follow up.

The second was delayed refer to our centers because of more frequent and long time conservative treatment in the previous centers which make ACL reconstruction susceptible to more complication rate. We could suggest further studies are required to understand with more sample size and biomechanical evaluation with this tool at a long-term follow-up to prove the KT efficacy in the ACL tear diagnosis.

\section{Acknowledgments}

None.

\section{Conflicts of interest}

None.

\section{References}

1. Freedman KB, D'Amato MJ, Nedeff DD et al. Arthroscopic anterior cruciate ligament reconstruction: a metaanalysis comparing patellar tendon and hamstring tendon autografts. Am J Sports Med. 2003:31(1):2-11.

2. Boden BP, Dean G, Feagin JA, Garrett WE Mechanisms of anterior cruciate ligament injury. Orthopedics 2000:23(6):573-578.

3. Feller JA, Webster KE A Randomized Comparison of Patellar Tendon and Hamstring Tendon Anterior Cruciate Ligament Reconstruction. Am J Sports Med. 2003:31(4):564-573.

4. Dutho VB, Barea C, Abrassart S et al. Anatomy of the anterior cruciate ligament. Knee Surg Sports Traumatol Arthrosc. 2006:14(3):204-213. 
5. Edwards A, Bull AM, Amis AA The attachments of the anteromedial and posterolateral fibre bundles of the anterior cruciate ligament part 1: tibial attachment. Knee Surg Sports Traumatol Arthrosc. 2008:16(1):29-36

6. Yamamoto Y, Hsu WH, Woo SL et al. A Comparison of a Lateral and an Anatomical Femoral Tunnel Placement Knee Stability and Graft Function After Anterior Cruciate Ligament Reconstruction. Am J Sports Med. 2004:32( 8):1825-1832.

7. Baquie PM, Brukner P Injuries Presenting to an Australian Sports Medicine Centre: A 12-Month Study. Clin J Sport Med. 1997:7(1):28-31.

8. Rayan F, Nanjayan SK, Quah C et al. Review of evolution of tunnel position in anterior cruciate ligament reconstruction. World J Orthop. 2015:6(2):252-262

9. Kaseta MK, DeFrate LE, Charnock BL et al. Reconstruction Technique Affects Femoral Tunnel Placement in ACL Reconstruction. Clin Orthop Relat Res. 2008:466(6):1467-1474.

10. Loh JC, Fukuda Y, Tsuda E et al. Knee stability and graft function following anterior cruciate ligament reconstruction: Comparison between 11 o'clock and 10 o'clock femoral tunnel placement. 2002 Richard O'Connor Award paper. Arthroscopy. 2003:19(3):297-304.

11. Khalfayan EE, Sharkey PF, Alexander AH et al. The relationship between tunnel placement and clinical results after anterior cruciate ligament reconstruction. Am J Sports Med. 1996:24(3):335-341.
12. Millett PJ, Willis AA, Warren RF Associated injuries in pediatric and adolescent anterior cruciate ligament tears: Does a delay in treatment increase the risk of meniscal tear. Arthroscopy. 2002:18(9):955-959.

13. Frobell RB, Roos EM, Roos HP et al. A Randomized Trial of Treatment for Acute Anterior Cruciate Ligament Tears. $N$ Engl J Med. 2010:363(4):331-342.

14. Muller W Knee ligament injuries. Pathoanatomy, biomechanics, instabilities and possibilities of treatment in acute and chronic injuries. IntOrthop. 1996:20(4):266-270.

15. Mae T, Shino K, Miyama T et al. Single- versus two-femoral socket anterior cruciate ligament reconstruction technique: Biomechanical analysis using a robotic simulator. Arthroscopy. 2001:17(7):708-716.

16. Steadman J, Mair S (1999) Anterior cruciate ligament reconstruction with bone-patellar tendon-bone autograft two-incision technique. Operative Techniques in Orthopedics 9(4): 273-280.

17. Kartus J, Magnusson L, Stener S, Brandsson S, Eriksson BI, et al. (1999) Complications following arthroscopic anterior cruciate ligament reconstruction. A 2-5-year follow-up of 604 patients with special emphasis on anterior knee pain. Knee Surg Sports Traumatol Arthrosc 7(1): 2-8.

18. Kannus P, Järvinen M (1987) Conservatively treated tears of the anterior cruciate ligament. Long-term results. J Bone Joint Surg Am 69(7): $1007-$ 1012. 